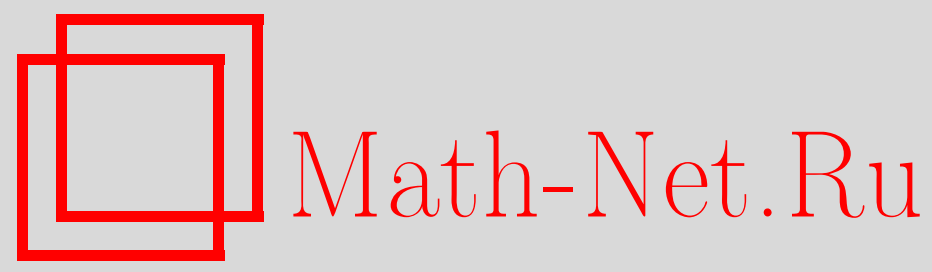

А. В. Устинов, О количестве слагаемых в асимптотической формуле для числа решений уравнения Варинга, Матем. заметки, 1998, том 64, выпуск 2, 285-296

DOI: https://doi.org/10.4213/mzm1396

Использование Общероссийского математического портала Math-Net.Ru подразумевает, что вы прочитали и согласны с пользовательским соглашением http://www.mathnet.ru/rus/agreement

Параметры загрузки:

IP : 3.85 .7 .115

26 апреля 2023 г., $15: 19: 52$

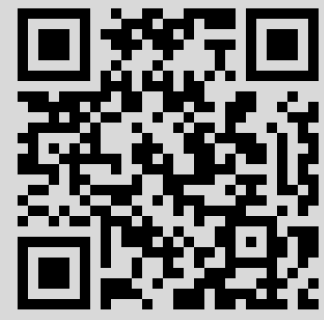


УДК 511.3

\section{О КОЛИЧЕСТВЕ СЛАГАЕМЫХ В АСИМПТОТИЧЕСКОЙ ФОРМУЛЕ ДЛЯ ЧИСЛА РЕШЕНИЙ УРАВНЕНИЯ ВАРИНГА}

\section{А. В. Устинов}

В статье получается оценка количества слагаемых в асимптотической формуле для числа решений уравнения Варинга. Это удается сделать благодаря рекуррентному процессу, даюшему лучшее понижение, чем теорема Виноградова о среднем.

Библиографоял: 3 названия.

1. Введение. Пусть $N_{k}^{(P)}\left(\lambda_{1}, \ldots, \lambda_{n}\right)$ - число решений системы

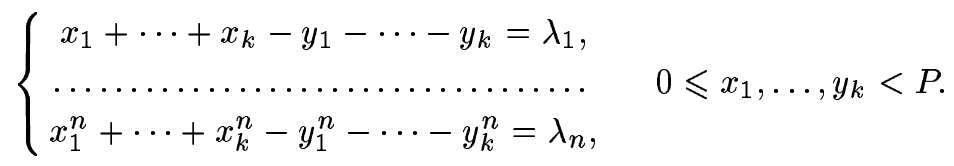

(Здесь и далее, переменные принимают только целые значения.) Согласно теореме Виноградова о среднем при $k \geqslant n \tau$ выполняется оценка

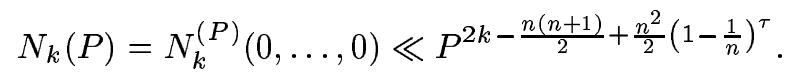

Обозначим через $I_{k}(P)$ число решений уравнения Харди

$$
x_{1}^{n}+\cdots+x_{k}^{n}-y_{1}^{n}-\cdots-y_{k}^{n}=0, \quad 0 \leqslant x_{1}, \ldots, y_{k}<P .
$$

Формула (1) позволяет получить оценку для $I_{k}(P)$

$$
\begin{aligned}
I_{k}(P) & \ll \sum_{\left|\lambda_{1}\right|<k P} \ldots \sum_{\left|\lambda_{n-1}\right|<k P^{n-1}} N_{k}^{(P)}\left(\lambda_{1}, \ldots, \lambda_{n-1}, 0\right) \\
& \ll P^{n(n-1) / 2} N_{k}(P) \ll P^{2 k-n+\frac{n^{2}}{2}\left(1-\frac{1}{n}\right)^{\tau} .}
\end{aligned}
$$

Пусть $I(N)$ - число решений уравнения Варинга

$$
x_{1}^{n}+\cdots+x_{k}^{n}=N, \quad 0 \leqslant x_{1}, \ldots, x_{k} \leqslant N^{1 / n} .
$$

В книге [1] доказано, что при $n \geqslant 4$ и $k \geqslant 2\left[n^{2}(2 \ln n+\ln \ln n+5)\right]$ справедлива асимптотическая формула

$$
I(N)=\sigma(N) \gamma N^{k / n-1}+O\left(N^{k / n-1-1 /(20 n \ln n)}\right),
$$

где $\gamma=(\Gamma(1+1 / n))^{k} / \Gamma(k / n), \sigma(N) \geqslant c_{0}(n, k)>0$.

$\mathrm{B}$ настоящей работе доказывается, что при $k \geqslant n(n-1)+n \tau$

$$
I_{k}(P) \ll P^{2 k-n+\frac{n}{2}\left(1-\frac{1}{n}\right)^{\tau}}
$$

Этот результат уточняет оценку (2), благодаря чему удается доказать, что формула (3) верна при $n \geqslant 4$ и $k \geqslant 2\left[n^{2}(\ln n+\ln \ln n+6)\right]$. 
2. Свойства коэффициентов Фурье некоторых функций. В работе [2] доказано следующее утверждение.

Лемма 1. Пусть $N_{1}, \ldots, N_{n}$ - неотрицательные иелые, $F\left(\alpha_{1}, \ldots, \alpha_{n}\right)$ - неотрицательная вещественная функиия, определенная на кубе $E_{n}=[0,1]^{n}$ и интегрируемая по Лебегу. Пусть также $c\left(\lambda_{1}, \ldots, \lambda_{n}\right)$ - коэффичиенты фурье $F\left(\alpha_{1}, \ldots, \alpha_{n}\right)-$ неотрицательные вещ,ественные числа. Тогда для любых иелых $\mu_{1}, \ldots, \mu_{n}$

$$
\sum_{\left|\lambda_{1}\right| \leqslant N_{1}} \cdots \sum_{\left|\lambda_{n}\right| \leqslant N_{n}} c\left(\lambda_{1}+\mu_{1}, \ldots, \lambda_{n}+\mu_{n}\right) \leqslant 4^{n} \sum_{\left|\lambda_{1}\right| \leqslant N_{1}} \ldots \sum_{\left|\lambda_{n}\right| \leqslant N_{n}} c\left(\lambda_{1}, \ldots, \lambda_{n}\right) .
$$

В дальнейших рассуждениях нам понадобятся леммы 2 и 3 , которые доказываются как лемма 1.

ЛЕмма 2. Пусть $N$ - неотрицательное действительное число, $a, b$ - иелье, $q$ - натуральное. Пусть также $F(\alpha)$ - неотрицательная вещественная функиия, представимая конечным рядом Фурье с неотрицательными коэффициентами $(\lambda)$. Тогда справедливы неравенства

$$
\sum_{|\lambda| \leqslant N} c(a \lambda+b) \leqslant 4 q \int_{0}^{1} F(\alpha) \Phi(\alpha) d \alpha \leqslant 4 q \sum_{|\lambda| \leqslant N q^{-1}} c(a q \lambda)
$$

$2 \partial e$

$$
\Phi(\alpha)=\sum_{|\lambda| \leqslant N q^{-1}}\left(1-\frac{|\lambda|}{\left[N q^{-1}\right]+1}\right) e^{-2 \pi i \alpha a q \lambda} \geqslant 0
$$

ДокАЗАтЕЛьСтво. Докажем лемму в случае $a=1$. При произвольном $a$ доказательство не изменится. Пусть $N_{1}=\left[N q^{-1}\right]+1$. Тогда

$$
\sum_{|\lambda| \leqslant N} c(\lambda+b) \leqslant \sum_{\mu=0}^{q-1} \sum_{|\lambda| \leqslant N_{1}} c(q \lambda+\mu+b)=\sum_{\mu=0}^{q-1} \sigma(\mu)
$$

где

$$
\sigma(\mu)=\sum_{|\lambda| \leqslant N_{1}} c(q \lambda+\mu+b)
$$

Оценим величину $\sigma(\mu)$ :

$$
\begin{aligned}
\sigma(\mu) & =\frac{1}{N_{1}^{2}} \sum_{x, y=1}^{N_{1}} \sum_{|\lambda+x-y| \leqslant N_{1}} c(q(\lambda+x-y)+\mu+b) \\
& \leqslant \frac{1}{N_{1}^{2}} \sum_{x, y=1}^{N_{1}} \sum_{|\lambda| \leqslant 2 N_{1}-1} c(q(\lambda+x-y)+\mu+b) \\
& =\frac{1}{N_{1}^{2}} \sum_{x, y=1}^{N_{1}} \sum_{|\lambda| \leqslant 2 N_{1}-1} \int_{0}^{1} F(\alpha) e^{-2 \pi i \alpha(q(\lambda+x-y)+\mu+b)} d \alpha \\
& =\frac{1}{N_{1}^{2}} \int_{0}^{1} F(\alpha)\left|\sum_{x=1}^{N_{1}} e^{-2 \pi i \alpha q x}\right|_{|\lambda| \leqslant 2 N_{1}-1}^{2} e^{-2 \pi i \alpha(q \lambda+\mu+b)} d \alpha .
\end{aligned}
$$


Оценивая последнюю сумму тривиально, получим

$$
\begin{aligned}
\sigma(\mu) & \leqslant \frac{4}{N_{1}} \int_{0}^{1} F(\alpha)\left|\sum_{x=1}^{N_{1}} e^{-2 \pi i \alpha q x}\right|^{2} d \alpha \\
& =\frac{4}{N_{1}} \int_{0}^{1} F(\alpha) \sum_{|\lambda| \leqslant N_{1}-1}\left(N_{1}-|\lambda|\right) e^{-2 \pi i \alpha q \lambda} d \alpha=4 \int_{0}^{1} F(\alpha) \Phi(\alpha) d \alpha .
\end{aligned}
$$

Отсюда имеем

$$
\sigma(\mu) \leqslant 4 \int_{0}^{1} F(\alpha) \Phi(\alpha) d \alpha=4 \sum_{|\lambda| \leqslant N_{1}-1}\left(1-\frac{|\lambda|}{N_{1}}\right) c(q \lambda) \leqslant 4 \sum_{|\lambda| \leqslant N q^{-1}} c(q \lambda) .
$$

Подставляя полученные неравенства в формулу (5), получим утверждение леммы.

СлЕДСТВИЕ. Пусть выполняются условия леммы $2 u \mathrm{Nq}^{-1}=O(1)$. Тогда справедлива оченка

$$
\sum_{|\lambda| \leqslant N q^{-1}} c(a q \lambda) \ll c(0)
$$

ДокАЗАТЕЛЬСтво. Подставляя в оценку (4) вместо $N, a, q, b$ величины $N q^{-1}, a q$, $N_{1}, 0$ соответственно, получим

$$
\sum_{|\lambda| \leqslant N q^{-1}} c(a q \lambda) \leqslant 4 N_{1} \sum_{|\lambda| \leqslant N\left(q N_{1}\right)^{-1}} c\left(a q N_{1} \lambda\right)=4 N_{1} c(0) \ll c(0) .
$$

Полностью аналогично доказываются лемма 3 и ее следствие.

ЛЕмма 3. Для любых иельх $a_{1,1}, a_{1,2}, \ldots, a_{n, n}, b_{1}, \ldots, b_{n}$ и натуральных $q_{1}, \ldots, q_{n}$ положим

$$
l_{\nu}=\sum_{j=1}^{n} a_{\nu, j} \lambda_{j}, \quad l_{\nu}^{\prime}=\sum_{j=1}^{n} a_{\nu, j} q_{j} \lambda_{j}, \quad \nu=1, \ldots, n .
$$

Предположим, далее, что $N_{1}, \ldots, N_{n}$ - неотрицательные действительные числа и $F\left(\alpha_{1}, \ldots, \alpha_{n}\right)$ - неотрицательная вещественная функиия, представимая конечным рядом Фурье с неотричательными коэффичиентами $c\left(\lambda_{1}, \ldots, \lambda_{n}\right)$. Тогда справедлива оченка

$$
\begin{aligned}
& \sum_{\left|\lambda_{1}\right| \leqslant N_{1}} \ldots \sum_{\left|\lambda_{n}\right| \leqslant N_{n}} c\left(l_{1}+b_{1}, \ldots, l_{n}+b_{n}\right) \\
& \leqslant 4^{n} q_{1} \cdots q_{n} \int_{0}^{1} \cdots \int_{0}^{1} F\left(\alpha_{1}, \ldots, \alpha_{n}\right) \Phi d \alpha_{1} \cdots d \alpha_{n} \\
& \leqslant 4^{n} q_{1} \cdots q_{n} \sum_{\left|\lambda_{1}\right| \leqslant N_{1} q_{1}^{-1}} \cdots \sum_{\left|\lambda_{n}\right| \leqslant N_{n} q_{n}^{-1}} c\left(l_{1}^{\prime}, \ldots, l_{n}^{\prime}\right),
\end{aligned}
$$

$2 \partial e$

$$
\begin{aligned}
\Phi= & \sum_{\left|\lambda_{1}\right| \leqslant N_{1} q_{1}^{-1}} \cdots \sum_{\left|\lambda_{n}\right| \leqslant N_{n} q_{n}^{-1}}\left(1-\frac{\left|\lambda_{1}\right|}{\left[N_{1} q_{1}^{-1}\right]+1}\right) \cdots\left(1-\frac{\left|\lambda_{n}\right|}{\left[N_{n} q_{n}^{-1}\right]+1}\right) \\
& \times e^{-2 \pi i\left(\alpha_{1} l_{1}^{\prime}+\cdots+\alpha_{n} l_{n}^{\prime}\right)} \geqslant 0 .
\end{aligned}
$$


СЛЕДСТВИЕ. Пусть выполняются условия леммы 3 и $N_{j} q_{j}^{-1}=O(1)$ при некотором $j, 1 \leqslant j \leqslant n$. Тогда если вычисления проводятся с точностью до констант, то мохнно считать, что $\lambda_{j}$ принимает только нулевое значение.

\section{3. Основные леммы.}

Лемма 4. Пусть $1 \leqslant r \leqslant n, H, Q, \mu_{r}, \ldots, \mu_{n-1}, a-$ челые числа, $H^{\prime}=H-a Q$. Тогда система

$$
\left\{\begin{array}{c}
x_{1}+\cdots-y_{k}=0, \\
\cdots \cdots \cdots \cdots \cdots \cdots \cdots \\
x_{1}^{r-1}+\cdots-y_{k}^{r-1}=0 \\
C_{n}^{r}\left(x_{1}^{r}+\cdots-y_{k}^{r}\right)=\mu_{r} Q \\
C_{n}^{r+1}\left(x_{1}^{r+1}+\cdots-y_{k}^{r+1}\right)+\mu_{r} H=\mu_{r+1} Q \\
\cdots \cdots \cdots \cdots \cdots \cdots \cdots \cdots \cdots \cdots \cdots \cdots \\
C_{n}^{n-1}\left(x_{1}^{n-1}+\cdots \cdots y_{k}^{n-1}\right)+\mu_{n-2} H=\mu_{n-1} Q \\
C_{n}^{n}\left(x_{1}^{n}+\cdots-y_{k}^{n}\right)+\mu_{n-1} H=\mu_{n}
\end{array}\right.
$$

равносильна системе

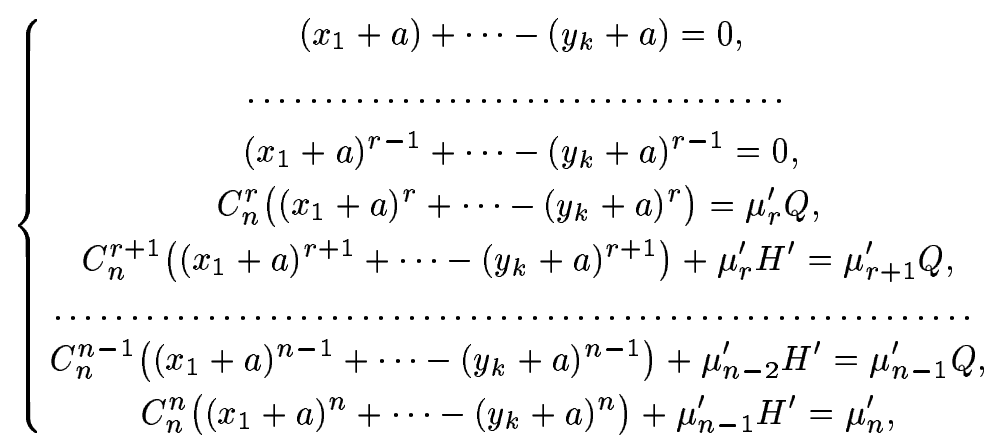

əde

$$
\mu_{l}^{\prime}=\sum_{j=r}^{l} C_{n-j-1}^{l-j} a^{l-j} \mu_{j}, \quad l=r, \ldots, n-1, \quad \mu_{n}^{\prime}=\mu_{n}
$$

ДокАЗАТЕЛЬСТво. Первые $r-1$ уравнений системы (8) непосредственно следуют из системы (7). Положим $\mu_{r-1}=0$. Пусть $r \leqslant l \leqslant n-1$. Тогда

$$
\begin{aligned}
C_{n}^{l} & \left(\left(x_{1}+a\right)^{l}+\cdots-\left(y_{k}+a\right)^{l}\right)+\mu_{l-1}^{\prime} H^{\prime}-\mu_{l}^{\prime} Q \\
= & C_{n}^{l} \sum_{j=r}^{l} C_{l}^{j} a^{l-j}\left(x_{1}^{j}+\ldots-y_{k}^{j}\right)+\mu_{l-1}^{\prime} H^{\prime}-\mu_{l}^{\prime} Q \\
= & \sum_{j=r}^{l} C_{n-j}^{l-j} a^{l-j}\left(\mu_{j} Q-\mu_{j-1} H\right)+(H-a Q) \sum_{j=r}^{l} C_{n-j-1}^{l-j-1} a^{l-j-1} \mu_{j} \\
& -Q \sum_{j=r}^{l} C_{n-j-1}^{l-j} a^{l-j} \mu_{j}
\end{aligned}
$$




$$
\begin{aligned}
= & H \sum_{j=r}^{l}\left(C_{n-j-1}^{l-j-1} a^{l-j-1} \mu_{j}-C_{n-j}^{l-j} a^{l-j} \mu_{j-1}\right) \\
& +Q \sum_{j=r}^{l} a^{l-j} \mu_{j}\left(C_{n-j}^{l-j}-C_{n-j-1}^{l-j-1}-C_{n-j-1}^{l-j}\right)=0 .
\end{aligned}
$$

При $l=n$ и в обратную сторону доказательство проводится аналогично.

ЗАмЕчАнИЕ. Если в системе (7) величины $\mu_{r}, \ldots, \mu_{n-1}$ независимо друг от друга пробегают целые числа, то в системе (8) величины $\mu_{r}^{\prime}, \ldots, \mu_{n-1}^{\prime}$ также независимо друг от друга пробегают целые числа.

Лемма 5. Пусть $1 \leqslant r \leqslant n, H, Q, \beta-$ челые числа, $0 \leqslant \beta \leqslant r+1, p-$ простое, $(Q, p)=1, H=p^{\alpha} h,(h, p)=1, \gamma=\min (1, \alpha)$ и $\alpha \beta=0$. Тогда система

$$
\left\{\begin{array}{c}
x_{1}+\cdots-y_{k}=0 \\
\cdots \cdots \cdots \cdots \cdots \cdots \\
x_{1}^{r-1}+\cdots-y_{k}^{r-1}=0 \\
C_{n}^{r} p^{r}\left(x_{1}^{r}+\cdots-y_{k}^{r}\right)=\mu_{r} Q p^{\beta} \\
\cdots \cdots \cdots \cdots \cdots \cdots \cdots \cdots \cdots \cdots \cdots \\
C_{n}^{n} p^{n}\left(x_{1}^{n}+\cdots \cdots y_{k}^{n}\right)+\mu_{n-1} H p^{\beta}=0
\end{array}\right.
$$

равносильна системе

$$
\left\{\begin{array}{c}
x_{1}+\cdots-y_{k}=0 \\
\ldots \ldots \cdots \cdots \cdots \cdots \\
x_{1}^{r-1}+\cdots-y_{k}^{r-1}=0 \\
C_{n}^{r}\left(x_{1}^{r}+\cdots-y_{k}^{r}\right)=\lambda_{r} Q p^{1-\gamma} \\
\ldots \cdots \cdots \cdots \cdots \cdots \cdots \cdots \cdots \\
C_{n}^{n}\left(x_{1}^{n}+\cdots-y_{k}^{n}\right)+\lambda_{n-1} H p^{-\gamma}=0
\end{array}\right.
$$

где величины $\mu_{r}, \ldots, \mu_{n-1} u \lambda_{r}, \ldots, \lambda_{n-1}$ связаны равенствами

$$
\mu_{r}=p^{(r+1)-\beta-\gamma} \lambda_{r}, \quad \ldots, \quad \mu_{n-1}=p^{n-\beta-\gamma} \lambda_{n-1} .
$$

ДокАЗАТЕЛЬСтво. Из равенств (9) следует, что величины $\mu_{r}, \ldots, \mu_{n-1}$ должны иметь вид (11). После подстановки этих выражений в систему (9) и сокращений, получим систему (10). Обратные рассуждения доказьвают, что из равенств (10) следует справедливость системы (9).

Лемма 6. Рассмотрим систему сравнений

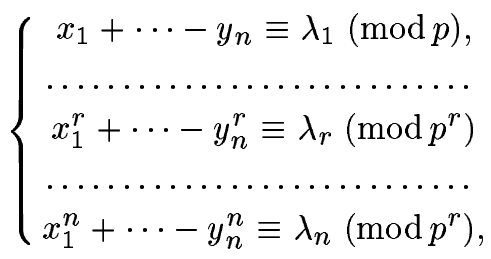

$$
0 \leqslant x_{1}, \ldots, y_{n} \leqslant N p^{r}-1
$$
$x_{s} \not \equiv x_{t}(\bmod p), \quad s \neq t, \quad 1 \leqslant s, t \leqslant n, \quad p \geqslant 4 n$. 
Если $T\left(\lambda_{1}, \ldots, \lambda_{n}\right)$ - число решений этой системы, то имеют место неравенства

$$
\frac{4}{9} N^{2 n} p^{r n+r(r-1) / 2} \leqslant T=T(0, \ldots, 0) \leqslant n ! N^{2 n} p^{r n+r(r-1) / 2} .
$$

Доказательство см. в [3].

4. О числе решений уравнения Харди и о проблеме Варинга. Определим натуральные числа $P_{0}, P_{1}, \ldots, P_{n-1}$ равенствами

$$
P_{0}=P, \quad P_{1}=\left[P_{0}^{1 / 2}\right]+1, \quad \ldots, \quad P_{n-1}=\left[P_{n-2}^{(n-1) / n}\right]+1 .
$$

Выберем простые $p_{1}, \ldots, p_{n-1}$ так, чтобы вьполнялись неравенства

$$
P_{0}^{1 / 2} \leqslant p_{1}<2 P_{0}^{1 / 2}, \quad P_{1}^{1 / 3} \leqslant p_{2}<2 P_{1}^{1 / 3}, \quad \ldots, \quad P_{n-2}^{1 / n} \leqslant p_{n-1}<2 P_{n-2}^{1 / n} .
$$

При $\nu=1, \ldots, n-1$ верны соотношения

$$
p_{\nu} P_{\nu} \geqslant P_{\nu-1}, \quad P_{\nu} \asymp P^{1 /(\nu+1)} \asymp p_{\nu}^{\nu}, \quad p_{1} \cdots p_{\nu} \asymp P^{1-1 /(\nu+1)} \asymp P_{\nu}^{\nu} .
$$

Пусть $0 \leqslant r \leqslant n-1$. Положим $Q_{r}=p_{1} \cdots p_{r}\left(Q_{0}=1\right)$. Обозначим через $T_{k, r}\left(P^{\prime}\right)$ число решений системы

$$
\left\{\begin{array}{c}
x_{1}+\cdots-y_{k}=0 \\
\cdots \cdots \cdots \cdots \cdots \\
x_{1}^{r}+\cdots-y_{k}^{r}=0 \\
C_{n}^{r+1}\left(x_{1}^{r+1}+\cdots-y_{k}^{r+1}\right)=\mu_{r+1} Q_{r} \\
C_{n}^{r+2}\left(x_{1}^{r+2}+\cdots-y_{k}^{r+2}\right)+\mu_{r+1} z_{r}=\mu_{r+2} Q_{r} \\
\left.\cdots \cdots \cdots \cdots \cdots \cdots \cdots \cdots \cdots \cdots \cdots \cdots y_{k}^{n-1}\right)+\mu_{n-2} z_{r}=\mu_{n-1} Q_{r} \\
C_{n}^{n-1}\left(x_{1}^{n-1}+\cdots \cdots y_{k}^{n}\right)+\mu_{n-1} z_{r}=0 \\
C_{n}^{n}\left(x_{1}^{n}+\cdots\right. \\
0 \leqslant x_{1}, \ldots, y_{k}<P^{\prime}, \quad 0 \leqslant z_{r}<Q_{r}
\end{array}\right.
$$

в которой $\mu_{r+1}, \ldots, \mu_{n-1}$ могут принимать произвольные целые значения. При $r=0$ получим равенство $T_{k, 0}\left(P_{0}\right)=I_{k}(P)$.

TEOPEMA 1. Пусть $n \geqslant 2, k \geqslant n^{2} / 2,1 \leqslant r \leqslant n-1, p_{r} \geqslant 4 n$. Тогда

$$
T_{k, r-1}\left(P_{r-1}\right) \ll p_{r}^{2(k-n)-1-r(r+1) / 2} P_{r-1}^{2 n} T_{k-n, r}\left(P_{r}\right) .
$$

ДокАЗАТЕЛЬСТво. Будем обозначать интеграл по единичному $n$-мерному кубу следуюшим образом:

$$
\int_{0}^{1} \cdots \int_{0}^{1} F\left(\alpha_{1}, \ldots, \alpha_{n}\right) d \alpha_{1} \cdots d \alpha_{n}=\int_{E_{n}} F\left(\alpha_{1}, \ldots, \alpha_{n}\right) d \bar{\alpha} .
$$

Пусть

$$
\begin{gathered}
f(x)=\alpha_{1} x+\cdots+\alpha_{r-1} x^{r-1}+\alpha_{r} C_{n}^{r} x^{r}+\cdots+\alpha_{n} C_{n}^{n} x^{n}, \\
S(a)=\sum_{x=0}^{P_{r}-1} e^{2 \pi i f\left(a+p_{r} x\right)}, \quad S=\sum_{a=0}^{p_{r}-1} S(a) .
\end{gathered}
$$


Согласно лемме 3

$$
T_{k, r-1}\left(P_{r-1}\right) \leqslant T_{k, r-1}\left(p_{r} P_{r}\right) \ll \sum_{z_{r-1}=0}^{Q_{r-1}-1} \int_{E_{n}}|S|^{2 k} \Phi d \bar{\alpha},
$$

где

$$
\Phi=\sum_{\mu_{r}, \ldots, \mu_{n-1}}\left(1-\frac{\left|\mu_{r}\right|}{N_{r}}\right) \cdots\left(1-\frac{\left|\mu_{n-1}\right|}{N_{n-1}}\right) e^{-2 \pi i\left(\alpha_{r} \mu_{r} Q_{r-1}+\cdots+\alpha_{n}\left(-\mu_{n-1} z_{r-1}\right)\right)} \geqslant 0
$$

с некоторыми неотрищательными $N_{r}, \ldots, N_{n-1}$. Положим $z_{r}=z_{r-1}+a Q_{r-1}$. Для каждого $z_{r-1}$ существует ровно одно значение $a$, для которого $\left(z_{r}, p_{r}\right)=p_{r}$. Обозначим это значение через $a_{0}$. При этом

$$
\begin{gathered}
|S|^{2 k}=\left.\left|S\left(a_{0}\right)+\sum_{\left.\substack{a \neq a_{0} \\
T_{k, r-1}\left(P_{r-1}\right)}(a)\right|^{2 k}} \ll\right| S\left(a_{0}\right)\right|^{2 k}+\mid \sum_{1}+T_{2},
\end{gathered}
$$

где

$$
T_{1}=\sum_{z_{r-1}} \int_{E_{n}}\left|S\left(a_{0}\right)\right|^{2 k} \Phi d \bar{\alpha}, \quad T_{2}=\sum_{z_{r-1}} \int_{E_{n}}\left|\sum_{a \neq a_{0}} S(a)\right|^{2 k} \Phi d \bar{\alpha} .
$$

Расписьвая $\Phi$ в виде суммы, вынося суммирование за знак интеграла и отбрасывая множители вида

$$
\left(1-\frac{\left|\mu_{r}\right|}{N_{r}}\right) \cdots\left(1-\frac{\left|\mu_{n-1}\right|}{N_{n-1}}\right)
$$

получим, что величина $T_{1}$ не превосходит числа решений системы

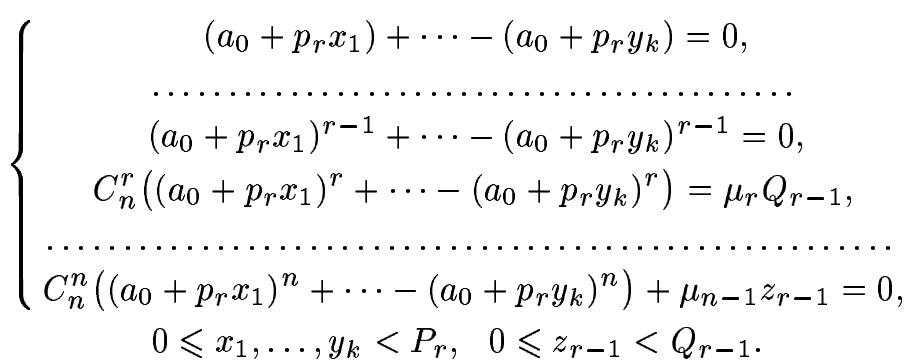

Применяя последовательно леммы 4 и 5 , получим, что $T_{1}$ не превосходит числа решений системы

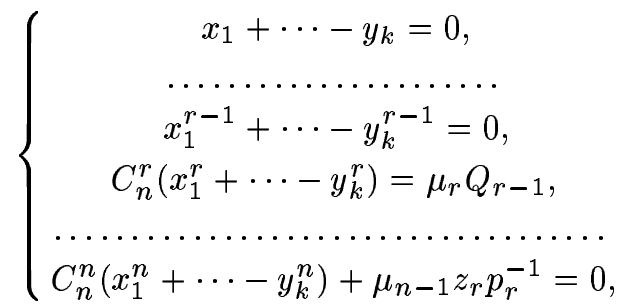

$$
\begin{aligned}
& 0 \leqslant x_{1}, \ldots, y_{k}<P_{r}, \quad z_{r}=z_{r-1}+a_{0} Q_{r-1}, \quad 0 \leqslant z_{r-1}<Q_{r-1} \text {. }
\end{aligned}
$$


Согласно лемме 3 имеем $T_{1} \ll p_{r}^{n-r} T_{3}$, где $T_{3}$ - число решений системы

$$
\left\{\begin{array}{c}
x_{1}+\cdots-y_{k}=0, \\
\ldots \ldots \cdots \cdots \cdots \cdots \cdots \\
x_{1}^{r-1}+\cdots-y_{k}^{r-1}=0 \\
C_{n}^{r}\left(x_{1}^{r}+\cdots-y_{k}^{r}\right)=\mu_{r} Q_{r}, \\
\cdots \cdots \cdots \cdots \cdots \cdots \cdots \\
C_{n}^{n}\left(x_{1}^{n}+\cdots-y_{k}^{n}\right)+\mu_{n-1} z_{r}=0
\end{array}\right.
$$

при прежних ограничениях на переменные. Так как $p_{1} \cdots p_{r} \asymp P_{r}^{r}$, то $\mu_{r}=O(1)$ и согласно следствию леммы 3 можно считать, что $\mu_{r}=0$. Отбрасьвая условие $\left(z_{r}, p_{r}\right)=p_{r}$, получим соотношение

$$
T_{1} \ll p_{r}^{n-r} T_{k, r}\left(P_{r}\right) \ll p_{r}^{n-r} P_{r}^{2 n} T_{k-n, r}\left(P_{r}\right)
$$

Оценим теперь величину $T_{2}$. Преобразуем подынтегральное выражение, фигурирующее в определении $T_{2}$ :

$$
\left|\sum_{a \neq a_{0}} S(a)\right|^{2 k}=\left|\sum_{a_{1} \neq a_{0}} \cdots \sum_{a_{k} \neq a_{0}} S\left(a_{1}\right) \cdots S\left(a_{k}\right)\right|^{2} .
$$

Будем относить набор $a_{1}, \ldots, a_{k}$ к первому классу, если среди чисел $a_{1}, \ldots, a_{k}$ можно найти $n$ различных. Все остальные наборы отнесем ко второму классу. Разобьем кратную сумму, стоящую в правой части равенства (16), на две суммы $\sigma_{1}$ и $\sigma_{2}$ по наборам первого и второго классов соответственно. При этом

$$
\begin{gathered}
\left|\sum_{a \neq a_{0}} S(a)\right|^{2 k}=\left|\sigma_{1}+\sigma_{2}\right|^{2} \ll\left|\sigma_{1}\right|^{2}+\left|\sigma_{2}\right|^{2}, \\
T_{2} \ll T_{4}+T_{5},
\end{gathered}
$$

где

$$
T_{4}=\sum_{z_{r-1}} \int_{E_{n}}\left|\sigma_{1}\right|^{2} \Phi d \bar{\alpha}, \quad T_{5}=\sum_{z_{r-1}} \int_{E_{n}}\left|\sigma_{2}\right|^{2} \Phi d \bar{\alpha} .
$$

Каждое слагаемое в сумме по наборам первого класса можно переписать так, чтобы $n$ различных чисел из набора $a_{1}, \ldots, a_{k}$ стояли на первых $n$ местах. Поэтому

$$
\begin{aligned}
T_{4} & \ll \sum_{z_{r-1}} \int_{E_{n}}\left|\sum_{a_{1}, \ldots, a_{n}}^{*} S\left(a_{1}\right) \cdots S\left(a_{n}\right)\right|^{2}\left|\sum_{a \neq a_{0}} S(a)\right|^{2(k-n)} \Phi d \bar{\alpha} \\
& \ll p_{r}^{2(k-n)-1} \sum_{z_{r-1}} \int_{E_{n}}\left|\sum_{a_{1}, \ldots, a_{n}}^{*} S\left(a_{1}\right) \cdots S\left(a_{n}\right)\right|^{2} \sum_{a \neq a_{0}}|S(a)|^{2(k-n)} \Phi d \bar{\alpha},
\end{aligned}
$$

где в сумме $\sum^{*}$ суммирование ведется по таким наборам $a_{1}, \ldots, a_{n}$, в которых $a_{s} \neq a_{t}$ при $s \neq t, 1 \leqslant s, t \leqslant n$. Поступая с функцией $\Phi$, как и раньше, получим, что

$$
T_{4} \ll p_{r}^{2(k-n)-1} T_{6},
$$


где $T_{6}$ - число решений системы

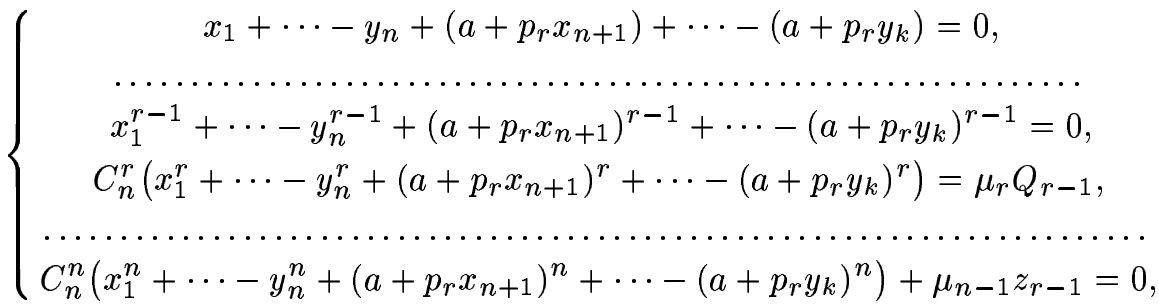

$$
\begin{aligned}
& 0 \leqslant x_{1}, \ldots, y_{n}<p_{r} P_{r}, \quad x_{s} \not \equiv x_{t}\left(\bmod p_{r}\right), \quad y_{s} \not \equiv y_{t}\left(\bmod p_{r}\right) \text { при } s \neq t \text {, } \\
& 0 \leqslant x_{n+1}, \ldots, y_{k}<P_{r}, \quad 0 \leqslant a<p_{r}, \quad a \neq a_{0}, \quad 0 \leqslant z_{r-1}<Q_{r-1} \text {. }
\end{aligned}
$$

По лемме 3

$$
T_{6} \ll p_{r}^{(r+1)(n-r)} T_{7},
$$

где $T_{7}$ - число решений системы

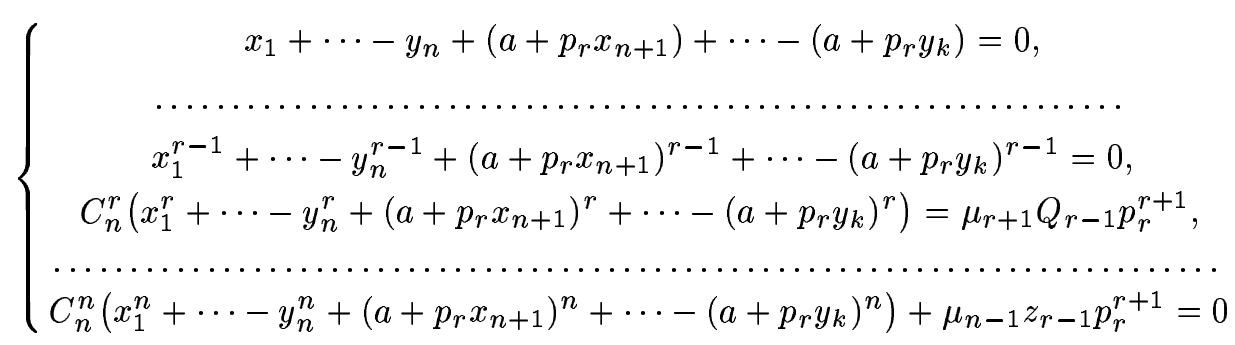

при прежних ограничениях на переменные. Согласно лемме 4 последнюю систему можно переписать в виде

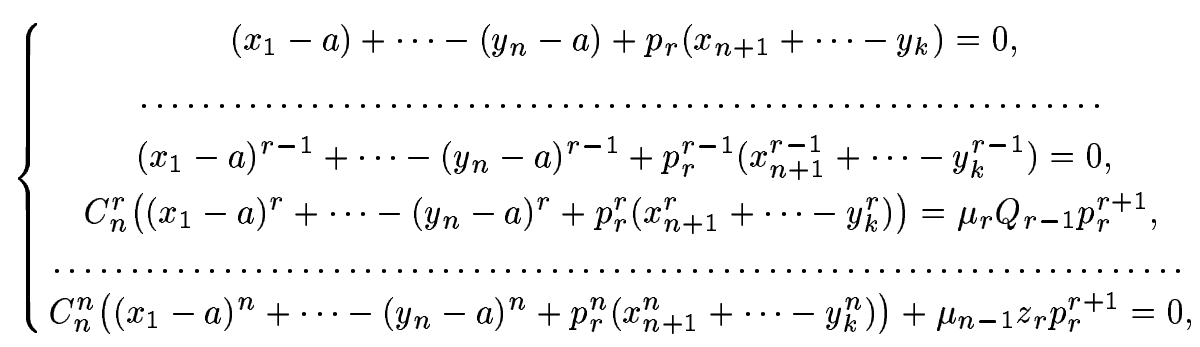

где $z_{r}=z_{r-1}+a Q_{r-1}$ и переменные меняются в прежних пределах. Обозначим через $N_{n}^{*}\left(\lambda_{1}, \ldots, \lambda_{n}\right)$ число решений системы

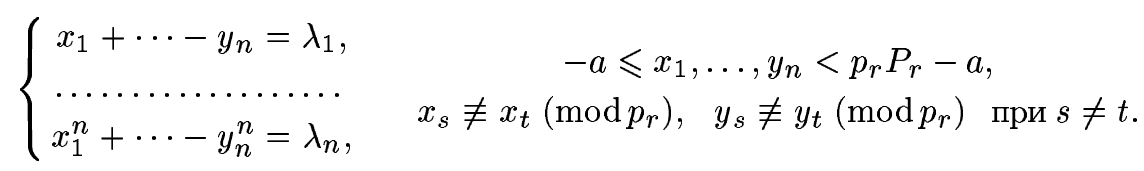

При этом величину $T_{7}$ можно записать в виде

$$
T_{7}=\sum_{\lambda_{1}, \ldots, \lambda_{n}} N_{n}^{*}\left(\lambda_{1} p_{r}, \ldots, \lambda_{r+1} p_{r}^{r+1}, \ldots, \lambda_{n} p_{r}^{r+1}\right) T_{8}\left(\lambda_{1} p_{r}, \ldots, \lambda_{r+1} p_{r}^{r+1}, \ldots, \lambda_{n} p_{r}^{r+1}\right)
$$


где $T_{8}\left(\lambda_{1}, \ldots, \lambda_{n}\right)$ - число решений системы

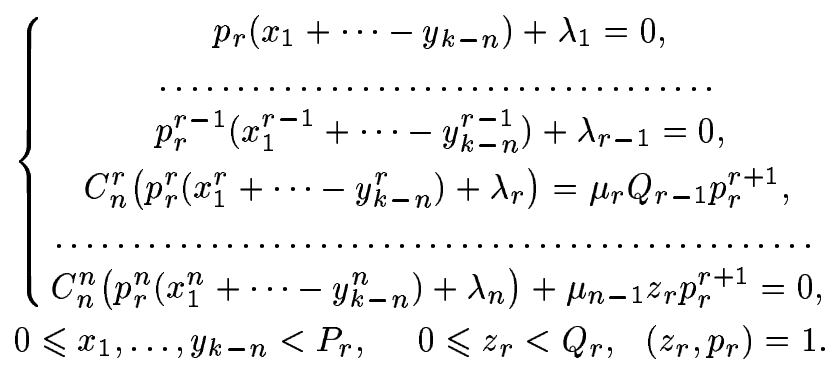

Пусть $T_{8}=T_{8}(0, \ldots, 0)$. В силу леммы 6

$T_{7} \leqslant T_{8} \sum_{\lambda_{1}, \ldots, \lambda_{n}} N_{n}^{*}\left(\lambda_{1} p_{r}, \ldots, \lambda_{r+1} p_{r}^{r+1}, \ldots, \lambda_{n} p_{r}^{r+1}\right) \ll P_{r-1}^{2 n} p_{r}^{-r(r+1) / 2-(r+1)(n-r)} T_{8}$.

Согласно лемме 5 величина $T_{8}$ не превосходит числа решений системы

$$
\left\{\begin{array}{c}
x_{1}+\cdots-y_{k-n}=0 \\
\cdots \cdots \cdots \cdots \cdots \cdots \\
x_{1}^{r-1}+\cdots-y_{k-n}^{r-1}=0 \\
C_{n}^{r}\left(x_{1}^{r}+\cdots-y_{k-n}^{r}\right)=\mu_{r} Q_{r} \\
\cdots \cdots \cdots \cdots \cdots \cdots \cdots \cdots \cdots \\
C_{n}^{n}\left(x_{1}^{n}+\cdots \cdots y_{k-n}^{n}\right)+\mu_{n-1} z_{r}=0
\end{array}\right.
$$

при прежних ограничениях на переменные. Так как $p_{1} \cdots p_{r} \asymp P_{r}^{r}$, то $\mu_{r}=O(1)$. Согласно следствию из леммы 3 можно считать, что $\mu_{r}=0$. Отбрасывая условие $\left(z_{r}, p_{r}\right)=1$, получим неравенство

$$
T_{8} \ll T_{k-n, r}\left(P_{r}\right)
$$

Объединяя оценки (18)-(21), имеем

$$
T_{4} \ll p_{r}^{2(k-n)-1-r(r+1) / 2} P_{r-1}^{2 n} T_{k-n, r}\left(P_{r}\right) .
$$

Оценим величину $T_{5}$. Число наборов второго класса не превосходит $n^{k} p_{r}^{n-1}$. Поступая с функцией $\Phi$, как и раньше, получим, что

$$
\begin{aligned}
T_{5} & \ll p_{r}^{2 n-2} \sum_{z_{r-1}} \sum_{a \neq a_{0}} \int_{E_{n}}|S(a)|^{2 k} \Phi d \bar{\alpha} \\
& \ll p_{r}^{2 n-2} P_{r}^{2 n} \sum_{z_{r-1}} \sum_{a \neq a_{0}} \int_{E_{n}}|S(a)|^{2(k-n)} \Phi d \bar{\alpha} \ll p_{r}^{2 n-2} P_{r}^{2 n} T_{9},
\end{aligned}
$$


где $T_{9}$ - число решений системы

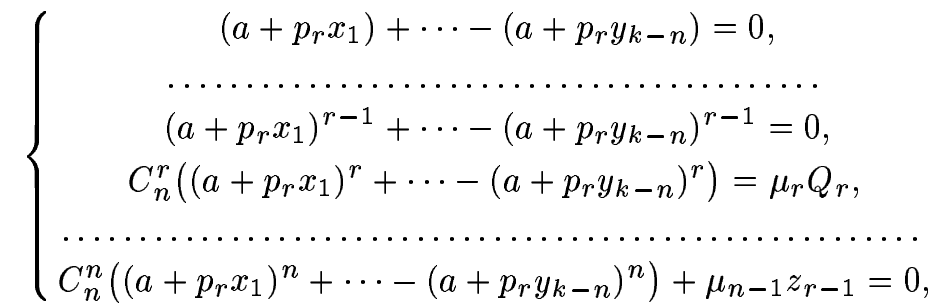

$$
\begin{aligned}
& 0 \leqslant x_{1}, \ldots, y_{k-n}<P_{r}, \quad 0 \leqslant z_{r-1}<Q_{r-1}, \quad 0 \leqslant a<p_{r}, \quad a \neq a_{0} \text {. }
\end{aligned}
$$

Применяя последовательно леммы 4 и 5 , получим, что $T_{9}$ не превосходит числа решений системы

$$
\left\{\begin{array}{c}
x_{1}+\cdots-y_{k-n}=0, \\
\cdots \cdots \cdots \cdots \cdots \cdots \\
x_{1}^{r-1}+\cdots-y_{k-n}^{r-1}=0 \\
C_{n}^{r}\left(x_{1}^{r}+\cdots-y_{k-n}^{r}\right)=\mu_{r} Q_{r} \\
\ldots \ldots \ldots \cdots \cdots \cdots \\
C_{n}^{n}\left(x_{1}^{n}+\cdots \cdots y_{k-n}^{n}\right)+\mu_{n-1} z_{r}=0
\end{array}\right.
$$

при прежних ограничениях на переменные. Снова заменяя $\mu_{r}$ на 0 , получим оценки

$$
T_{9} \ll T_{k-n, r}\left(P_{r}\right), \quad T_{5} \ll p_{r}^{2 n-2} P_{r}^{2 n} T_{k-n, r}\left(P_{r}\right) .
$$

Из оценок (15), (22) и (23) следует, что

$$
T_{k, r-1}\left(P_{r-1}\right) \ll\left(p_{r}^{n-r} P_{r}^{2 n}+p_{r}^{2(k-n)-1-r(r+1) / 2} P_{r-1}^{2 n}+p_{r}^{2 n-2} P_{r}^{2 n}\right) T_{k-n, r}\left(P_{r}\right)
$$

Так как $k \geqslant n^{2} / 2$, в формуле (24) главным членом в скобках является второе слагаемое. Поэтому

$$
T_{k, r-1}\left(P_{r-1}\right) \ll p_{r}^{2(k-n)-1-r(r+1) / 2} P_{r-1}^{2 n} T_{k-n, r}\left(P_{r}\right) .
$$

ТЕОрема 2. Пусть $n \geqslant 2, k \geqslant n^{2} / 2$ и выполняется оценка

$$
N_{k}(P) \ll P^{2 k-n(n+1) / 2+\varepsilon_{0}} .
$$

Тогда при $k_{1} \geqslant n(n-1)+k$ справедлива оченка

$$
I_{k_{1}}(P) \ll P^{2 k_{1}-n+\varepsilon_{0} / n}
$$

ДокАЗАТЕЛЬСтво. Теорему достаточно доказать при $k_{1}=n(n-1)+k$. Будем считать, что $P \geqslant(4 n)^{n^{2}}$. Это гарантирует вьполнение неравенства $p_{n-1} \geqslant 4 n$. Покажем, что для $\tau=0, \ldots, n-1$ верна оценка

$$
T_{k+n \tau, n-\tau-1}\left(P_{n-\tau-1}\right) \ll P_{n-\tau-1}^{2(k+n \tau)-n(n+1) / 2+\tau(\tau+1) / 2} p_{1} \cdots p_{n-\tau-1} P^{\varepsilon_{0} / n} .
$$


При $\tau=0$ надо оценить величину $T_{k, n-1}\left(P_{n-1}\right)$, равную числу решений системы

$$
\left\{\begin{array}{l}
x_{1}+\cdots-y_{k}=0 \\
\cdots \cdots \cdots \cdots \cdots \cdots \cdots \\
x_{1}^{n-1}+\cdots-y_{k}^{n-1}=0, \\
C_{n}^{n}\left(x_{1}^{n}+\cdots-y_{k}^{n}\right)=0
\end{array}\right.
$$

Переменная $z_{n-1}$ в системе не участвует, поэтому

$$
T_{k, n-1}\left(P_{n-1}\right) \ll N_{k}\left(P_{n-1}\right) p_{1} \cdots p_{n-1} \ll P_{n-1}^{2 k-n(n+1) / 2} p_{1} \cdots p_{n-1} P^{\varepsilon_{0} / n} .
$$

Пусть оценка (25) верна для некоторого $\tau, 0 \leqslant \tau \leqslant n-2$. Докажем ее справедливость при $\tau+1$. Согласно теореме 1

$T_{k+n(\tau+1), n-\tau-2}\left(P_{n-\tau-2}\right) \ll p_{n-\tau-1}^{2(k+n \tau)-1-\frac{(n-\tau-1)(n-\tau)}{2}} P_{n-\tau-2}^{2 n} T_{k+n \tau, n-\tau-1}\left(P_{n-\tau-1}\right)$.

Применяя неравенство (25), получим

$$
T_{k+n(\tau+1), n-\tau-2}\left(P_{n-\tau-2}\right) \ll P_{n-\tau-2}^{2(k+n(\tau+1))-\frac{n(n+1)}{2}+\frac{(\tau+1)(\tau+2)}{2}} p_{1} \cdots p_{n-\tau-2} P^{\varepsilon_{0} / n} .
$$

При $\tau=n-1$ формула (25) дает оценку

$$
I_{k_{1}}(P)=T_{k_{1}, 0}\left(P_{0}\right) \ll P_{0}^{2 k_{1}-n+\varepsilon_{0} / n} .
$$

СлЕДСТВИЕ. Пусть $n \geqslant 2, \tau \geqslant n / 2, k \geqslant n(n-1)+n \tau$. Тогда

$$
I_{k}(P) \ll P^{2 k-n+\frac{n}{2}\left(1-\frac{1}{n}\right)^{\tau}} .
$$

Доказательство непосредственно следует из теоремы 2 и оценки (1).

ЗАмЕчАниЕ. Тем жеметодом получаются оценки числа решений и асимптотические формулы для систем вида

$x_{1}^{\nu}+\cdots-y_{k}^{\nu}=0, \quad 0 \leqslant x_{1}, \ldots, y_{k}<P, \quad \nu=r_{1}, r_{2}, \ldots, r_{s}, \quad 1 \leqslant r_{1}<r_{2}<\cdots<r_{s}=n$. Так, например, для величины $I_{k, r, n}(P)$, равной числу решений системы

$$
\left\{\begin{array}{l}
x_{1}^{r}+\cdots-y_{k}^{r}=0, \\
x_{1}^{n}+\cdots-y_{k}^{n}=0,
\end{array} \quad 0 \leqslant x_{1}, \ldots, y_{k}<P,\right.
$$

при $n \geqslant 2, \tau \geqslant n / 2, k \geqslant n(n-1)+n \tau$ справедлива оценка

$$
I_{k, n, r}(P) \ll P^{2 k-r-n+\frac{r n}{2}\left(1-\frac{1}{n}\right)^{\tau}} .
$$

Теорема 3. Пусть $n \geqslant 4$. Тогда асимптотическая формула (3) справедлива nрu $k \geqslant 2\left[n^{2}(\ln n+\ln \ln n+6)\right]$.

Доказательство проводится аналогично доказательству теоремы 1 из [1, гл. 6] с заменой (1) при оценке интеграла по области второго класса на (27).

Автор благодарит Н. М. Коробова за поставленную задачу и внимательное руководство.

\section{СПИСОК ЦИТИРОВАННОЙ ЛИТЕРАТУРЫ}

[1] Виноградов И. М. Метод тригонометрических сумм в теории чисел. М.: Наука, 1980.

[2] Быковский В. А. О системах неравенств // Записки научн. семин. ЛОМИ. 1981. Т. 129. C. 3-33.

[3] Карацуба А. А. О системах сравнений // Изв. АН СССР. Сер. матем. 1965. Т. 29. C. 935-944. 